\title{
Sexualité violente, désir furieux : entre diction et indicible
}

\author{
NoÉMIE LABROUSSE \\ UnIVERSITÉ Aix-MARSEILle
}

Résumé : En prenant appui sur les textes d'Anne Hébert, sur son écriture, et en analysant les structures phrastiques autant que les interstices typographiques, cet article mettra en évidence une sexualité masculine, violente et bestiale, ébranlée par une fureur féminine, latente et silencieuse. Il s'agira de montrer cette cohabitation et de faire ressortir comment la sensualité désirée par les femmes tend à annihiler progressivement mais avec violence la sexualité jusque-là imposée par les hommes.

Mots-clés : Désir, Sexualité, Violence, Émancipation, Indicible.

Les chambres de bois, Kamouraska et Les fous de Bassan d'Anne Hébert dépeignent des relations homme-femme passionnées, violentes, voire mortelles. Ces relations humaines sont aussi le reflet des relations au sein d'une société patriarcale sur le déclin mais toujours présente et pesante. Ce règne de l'Homme influe sur le désir des personnages, sur leurs sexualités et sur les façons d'en parler dans les œuvres hébertiennes. L'intérêt sera de montrer comment les œuvres oscillent textuellement, stylistiquement, linguistiquement, grammaticalement entre le silence et la violence de la parole et comment elles passent d'un désir souterrain à une sexualité impudique. Le lecteur assiste ainsi à une transformation par la parole : de la soumission à une forme d'émancipation sexuelle et sensuelle.

\section{Des personnages brimés ou orientés}

Les romans mettent en scène des êtres brimés, qui sont tous, d'une façon ou d'une autre, sous le joug d'une instance supérieure. Comme déformés par trop de soumission, par trop de brimades, les personnages semblent avoir assimilé et être obligés de reproduire à l'infini des schémas de domination violents jusque dans leur sexualité. Cette sexualité codifiée par des instances autoritaires surgit dans les romans 
de façon violente entre provocation morale et dénonciation de ce qui se passe en coulisses.

\section{La société patriarcale}

La société dans laquelle évoluent les personnages dans les romans qui nous intéressent est un patriarcat, un monde dans lequel l'homme est désigné comme le dominant, le décideur. De cette position de supériorité, découlent des comportements violents, excusés par une conception hiérarchisée des relations homme-femme. Une dichotomie entre l'homme et la femme habite l'œuvre d'Anne Hébert. Comme toute lutte qui n'est pas seulement morale, celle-ci laisse des traces : « Couchée dans mon petit lit, contre le lit de ma mère, j'oublie sa recommandation de fermer les yeux quand elle se déshabille. Je découvre des marques bleues sur ses bras et ses épaules. » (Les fous de Bassan, 1982 : 209) Ce règne du mâle est aussi règne du mal. La femme est à la merci de l'homme, sans recours face à lui « hirsut[e] et mauvais ». ( $\left.F B^{1}: 40\right)$ Qu'elle soit morale ou physique, la souffrance féminine ne peut pas être dite, libérée. Comme le propose Marilyn Randall dans son étude comparative du roman et du film Les fous de Bassan, « [s]i le film tait [...] la voix des femmes, c'est qu'il réalise un aspect clé du roman : les femmes n'ont pas de voix, voire de vie, dans la société patriarcale où elles évoluent. » (Randall, 2001 : 110) Ainsi la principale caractéristique de la société patriarcale est de maintenir la femme dans un état passif et silencieux. C'est en ce sens que l'on peut parler d'instance castratrice et passéiste. Donner la parole aux femmes signerait la fin du règne du mâle.

\section{La religion}

Dans Kamouraska et Les fous de Bassan, le monde dans lequel évoluent les personnages est empreint de religiosité. Puritanisme, omniprésence et omnipotence de la religion se disent dans ces deux romans. Les femmes qui se doivent de maintenir intact et virginal leur honneur sont constamment ramenées vers la honte du péché originel commis par la première femme, l'Ève primitive. Chaque femme porte sur ses épaules le poids et la responsabilité de cette faute originelle et doit par son comportement irréprochable tenter d'approcher le pardon. La femme est à la merci de cette épée de Damoclès : «La lame de rasoir aurait fort bien pu se trouver là, dans la chambre. Suspendue par un fil, au-dessus de mon lit, de toute éternité... » (Kamouraska, 1997 [1970] : 116) Elle se retrouve confrontée à une impossible conciliation des ordonnances religieuses. Donner la vie sans pour autant perdre sa virginité. La culpabilité liée à l'acte sexuel, qui est pourtant l'acte par lequel la procréation est

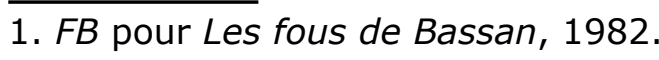


possible, pousse la femme à courir en vain après sa pureté natale. En quête de rédemption, la femme est esclave de la religion et de ses préceptes. Coincée, elle ne peut que se plier à cette loi religieuse, véritable loi du silence et du secret.

\section{L'héritage féminin}

La soumission féminine dans les romans étudiés ne tient pas qu'à la domination violente de la société patriarcale ou qu'à la mainmise culpabilisatrice de la religion. De par son statut de femme, le nouveau-né de sexe féminin est préparé dès sa naissance à prendre la relève et à perpétuer la lignée féminine. Enfermée dès sa venue au monde dans le monde clos et silencieux qu'est l'univers des femmes, la fille est marquée par une multitude de stéréotypes archaïques qui charrient les valeurs patriarcales. Sa personnalité et ses attentes s'en trouvent brimées et cela explique qu'aucune émancipation ne puisse être complète. Par mimétisme, la fille reproduira le schéma maternel hérité et transmis tel un secret de génération en génération.

Sous les chapeaux de paille leurs visages se rapprochent, chuchotent des histoires de naissance et de mort. Si je pouvais les écouter, avec leurs silences, de place en place, je saurais tout ce qu'une femme doit savoir. Mais je suis trop petite encore et l'ombre de l'amour qui surgit dans les conversations des mères est secrète et redoutable. (FB:115)

La divulgation de ce secret « redoutable » semble emprisonner davantage les femmes qui le connaissent. Car ce secret n'est finalement que la promesse d'obéissance à cet ordre patriarcal et à cette longue tradition de femmes soumises, femmes au foyer, ménagères bâillonnées : « $[\mathrm{M}]$ a sœur d'enfance qui est malheureuse et trop solennelle, depuis qu'elle a fait vœu d'obéissance à sa mère mourante. » (FB:122) Cette règle de soumission hiérarchisée place les femmes comme Catherine, Elisabeth, Olivia et Nora au bas de la pyramide sociale. La mère, la grand-mère, la tante ou toute autre initiatrice sont chargées d'enseigner à ces jeunes filles, encore des enfants, comment devenir femmes et survivre dans la société patriarcale. Ces figures féminines tutélaires, elles-mêmes conditionnées, sont devenues tout aussi oppressives et castratrices que les hommes ou la religion. Daniel Marcheix les désigne par l'appellation « mère mortifère ». (Marcheix, 2005 : 152) Mortifères, elles le sont dans le sens où elles castrent et privent de vie ${ }^{2}$ leur descendance.

\section{Un cheval de Troie narratif}

Tout particulièrement soumises à ces instances autoritaires, les femmes n'ont pas accès à la parole et ne peuvent pas exprimer leurs désirs. Puisque la parole n'est

2. Il ne s'agit pas ici de priver de vie en donnant la mort mais en empêchant d'exister. 
octroyée qu'aux représentants de l'ordre sociétal archaïque, c'est en fonction d'eux que les femmes décident d'agir. Ainsi, il s'agit pour elles de s'introduire au sein même des instances castratrices (société patriarcale, religion, monde féminin) afin d'engendrer une implosion. Pour Hubert Aquin, «tout désir, même celui de parler, est un désir de vivre. » (Aquin, 1997 : 50) C'est donc pour la revendication de son droit de vivre que la femme décide de prendre la parole, et ce, par tous les moyens. Mais ne pouvant accéder elle-même à la parole et forcée de renoncer à se faire entendre par sa propre voix, la femme s'insinue dans le discours de l'Autre. L'indicible auquel elle est soumise est ainsi détourné grâce à une phagocytose progressive de toutes les instances énonciatrices. C'est dans la voix de l'Autre et particulièrement dans la voix masculine de l'époux ou de l'oppresseur que la voix féminine se manifeste. Celui-là même qui maintient la femme dans un état passif, dans un statut d'objet, qui la prive de sa voix et qui lui impose le silence, est utilisé pour détruire son clan et les valeurs fondamentales de ce clan. «Cette corde est assez grande pour deux, Elisabeth, ma femme. Les liens du mariage, c'est ça. Une grosse corde bien attachée pour s'étouffer ensemble. Tu as promis pour le meilleur et pour le pire. » $\left(K^{3}: 86\right)$ La voix des hommes devient un outil de dénonciation de cette tradition reposant sur la violence masculine. L'ordre patriarcal est remis en question et renié de l'intérieur. Dès lors, les personnages de femmes n'auront de cesse de lutter contre la violence qui leur est imposée pour pouvoir dire leur désir.

\section{Une sexualité crue}

Malgré le joug des instances contraignantes, la sexualité se donne à voir et s'expose de façon très crue dans les romans d'Anne Hébert. Seul un langage délibérément violent, primitif et animal pouvait exprimer la domination masculine et la conception patriarcale des relations conjugales. La violence verbale et la crudité apparaissent aussi dans le discours des femmes qui, ayant enfin la possibilité d'évoquer leur désir, se retrouvent cependant sans langage pour le faire. N'ayant jamais eu droit à la parole, démunies, sans «langage-femmes » (Collin, $1976: 7$ ), selon I'expression de Françoise Collin, pour dire leur sexualité, elles empruntent aux hommes les mots qui leur manquent et amorcent une révolution tant textuelle que sexuelle. Elles parviennent à utiliser cette violence du verbe et du langage non seulement comme langue de désir, mais aussi comme outil de dénonciation du règne du mâle. 


\section{Une sexualité violente}

Dans les romans qui nous intéressent, les personnages masculins sont tous, d'une façon ou d'une autre, violents. Du plus éduqué au plus simple d'esprit, tous sont dépeints comme des prédateurs répondant à leurs plus bas instincts. Qu'ils soient mari, amant, cousin, oncle ou prêtre, tous ont en commun d'être des chasseurs, dont I'objectif est de dominer, de posséder, de blesser, de tuer. Leur omniprésence et leur omnipotence se concrétisent tout particulièrement dans la chambre conjugale lorsque : «De retour de chasse ils prennent leur femme dans le noir, sans enlever leurs bottes » $(F B: 40)$ ou encore lorsqu'« [i]l l'emmena sur le lit et la posséda avec maladresse et fureur. » (Les chambres de bois, 1996 [1958] : 98) La violence du désir masculin, véritable fureur, se métaphorise sous forme d'éléments naturels. Ainsi, dans Les fous de Bassan, la folie du vent renvoie inconsciemment à la folie de Stevens et, par un effet de transmission, le vent réfère à Stevens :

Je prétends que c'est la fureur de la tempête qui le possède et bat à grands coups sur toute sa peau tendue comme un tambour. (FB : 133 ; nous soulignons)

Je garde le souvenir confus d'une sorte d'ivresse s'emparant de moi, peu à peu, à force de contempler la mer démontée, me réduisant au rôle d'un fétu de paille emporté par la fièvre, tandis qu'une espèce de chant se formait dans mes veines en guise d'accompagnement à la fureur des éléments. (FB : 101-102; nous soulignons)

Le désir de I'homme est une tempête destructrice, un vent ravageur, une vague mortifère. « [E]lle retrouva en elle le ton de l'adoration de Michel qui montait, montait comme une vague pour la submerger. » $\left(C B^{4}: 122\right)$ Nombreux sont les éléments textuels confirmant cette violence et ce caractère mortifère de la sexualité et du désir masculins : «Cette fille est trop belle, il faudrait lui tordre le cou tout de suite, avant que... » (FB : 79) ; «Elle est si belle, cette femme, que je voudrais la noyer » $(C B: 75)$; « Me prendra de force, ne me lâchera que morte, dans une flaque de sang, comme une accouchée crevée. » ( $K: 163-164)$ Mais si les femmes semblent soumises à cette sexualité destructrice imposée par les hommes, elles recourent elles aussi à une énonciation crue et fortement sexualisée. 


\section{Traduire le désir féminin dans le langage-mâle : trahison ou jubilation?}

Pour ébranler la société patriarcale qui les brime, les femmes doivent prendre la parole et exprimer leurs désirs. Pour cela, elles font passer leur subjectivité par la voix de l'Autre et se réapproprient le vocable utilisé par cet Autre. La femme qui masculinise son vocabulaire et qui s'affirme en vient à adopter une perspective provocante et déstabilisatrice. Un renversement des rôles s'opère. La femme accède à la parole et du même coup au pouvoir : « J'ai ce pouvoir. Je suis Mme Rolland et je sais tout. [...] j'interviens [...] Je préside ». (K:123) Cependant, issue d'une longue lignée silencieuse qui n'avait pas à trouver de mots pour qualifier ses sentiments, la femme, au jour de la naissance de sa voix, se retrouve démunie. Sans mot pour définir ce qu'elle ressent, elle emprunte à I'homme son vocabulaire : « [J]'apprendrais tout de lui ». ( $F B: 216)$ De ce fait, la violence intègre le discours féminin. Amour et mort se trouvent liés dans cette parole féminine naissante mais jaillissant dans la fureur de se faire enfin entendre. La lutte primitive entre hommes et femmes se dit avec hargne : «Amour, amour, je te mords, je te bats, je te tue. » ( $K: 9)$ Au fur et à mesure des textes, on ne peut que remarquer les structures syntaxiques en miroir qui tendent à montrer cette symétrie s'établissant entre la parole de I'homme et la parole de la femme. Joutes visuelles et joutes syntaxiques sont omniprésentes et renvoient à la lutte des sexes : « Un homme et une femme côte à côte. Mari et femme. Se haïssent. Se provoquent mutuellement. » $(K: 131)$ Les relations hommefemme semblent ne pouvoir exister que dans la violence. Cette violence est défi, provocation, mais aussi séduction. Il s'agit de voir qui de l'homme ou de la femme sera le vainqueur de cette partie de chasse.

Stevens regardant Nora à présent. Nora regardant Stevens. (FB : 30)

Elle l'a regardé en plein visage. Elle a été regardée par lui en plein visage. $(F B: 215)$

Regarder tous les hommes, dans la rue. [...] Être regardée par eux. ( $K: 9)$

Regarde-moi un peu, car je vais te quitter pour toujours. ( $C B: 171)$

Les femmes se positionnent désormais face à l'homme, c'est-à-dire contre lui. L'homme prédateur n'est plus face à une proie mais face à une prédatrice en herbe. La femme se lance dans une traque amoureuse empruntée à I'homme : «Cette fois-ci c'est l'été et c'est moi "la chasseresse"» (FB: 126) ; «Leurs regards hardis sur "la chasseresse", comme ils m'appellent. » $(K: 65)$ Cette femme qui devient prédatrice récupère les termes employés par les hommes pour exprimer son propre désir : «Tu me devines, Antoine Tassy, et tu me traques, comme un bon chien de 
chasse. Et moi aussi je te flaire et je te découvre. » $(K: 66) ;$ «Mon cousin Stevens marche à quinze pas devant moi. Je me suis juré de le suivre à la trace jusqu'à ce qu'il se retourne de lui-même vers moi. » (FB: 127) Nora incarne parfaitement cette réappropriation exubérante du vocabulaire masculin. Mais son envie furieuse d'être l'égale de I'homme au niveau du langage met en danger le règne du mâle. Elspeth Tulloch affirme en ce sens :

Par ses rires cixousiens, son appropriation du vocabulaire masculin à l'encontre de Stevens, et son appel à la prudence lancé à Olivia au sujet de Stevens, Nora symbolise, dans la dernière scène du roman, la jeune femme moderne, libre sexuellement et contestataire - figure que Stevens, le patriarche en formation, ne peut ni accepter ni laisser vivre. (Tulloch, 2001: 96)

Nora en tant que menace pour la société patriarcale doit donc être sacrifiée.

Moi je prétends qu'à mesure que Nora m'injuriait et m'insultait, se grisant elle-même d'injures et d'insultes, le vocabulaire grossier des hommes de Griffin Creek, leur colère brutale, passant soudain par sa bouche de jeune fille, le coup de vent s'est levé sur la mer, au bout de l'horizon, entre cap Sec et cap Sauvagine. J'ai senti la menace de la tempête [...] cognant contre mes tempes, bien avant que rien ne soit visible dans le paysage, baigné de lune. ( $F B: 244)$

La masculinisation de la voix féminine entraîne un début d'inversion des valeurs. L'homme se voit dépossédé de son langage et conséquemment d'une partie de son pouvoir, car en face de lui se dresse désormais un être ayant les mêmes ressources que lui et pouvant le violenter verbalement à son tour.

D'ailleurs, loin de n'être que trahison des désirs féminins, cette langue crue semble offrir aux héroïnes une véritable jouissance verbale : « [S]e grisant elle-même d'injures et d'insultes ». (FB : 244) Chez Elisabeth, plus que chez toute autre héroïne hébertienne, la sexualité est violente, presque masculine. Elle est sûrement I'héroïne la plus ambiguë de toutes, souhaitant se défaire de la soumission de son époux Antoine Tassy, mais semblant trouver du plaisir dans la domination masculine : « Accéder à l'ombre du moindre désir de cet homme » $(K: 35)$, «[m]ais de nuit, je redeviens la complice d'Antoine. Jusqu'au dégoût le plus profond. La terreur la plus folle. » ( $K: 98)$ Elisabeth, ne semble pas savoir à quelle instance elle doit s'opposer et avec laquelle elle doit composer pour se libérer et accéder enfin à sa propre liberté, y compris à sa liberté sexuelle. Ici, alliée à Antoine, patriarche par excellence, elle s'oppose à la religion autant qu'à I'héritage féminin, mais l'isotopie reste empreinte de violence et de négativité. 
Je ris malgré ma crainte. Je suis sûre que mes petites tantes ne dorment pas et se signent en tremblant. Elles apprennent dans la nuit : l'ivresse, le blasphème, la violence, l'amour et la dérision.

La nuit, la Petite geint, parfois. De douleur ou de plaisir. Le crime est le même. ( $K: 98)$

La sexualité qu'elle semble revendiquer se partage entre plaisir et souffrance : «Vêtements lourds brusquement ouverts sur la tendresse du ventre. Comme une bête que I'on écorche. » $(K: 140)$ On peut parler d'un certain plaisir procuré par la violence verbale.

La différence est que les femmes font de cette violence un jeu sexuel, un jeu de séduction, tandis que les hommes perdent le contrôle et vont jusqu'au meurtre. L'œuvre hébertienne et les trois romans du corpus gravitent autour de la thématique du désir et des rapports homme/femme inscrits dans une relation dominant/ dominé(e) violente. Il n'est pas surprenant de constater que dans plusieurs textes s'établissent un champ lexical du désir ainsi qu'un champ lexical de la violence qui s'entrelacent pour faire sens. Reflets de la tragédie qui se joue dans ces romans, ces champs lexicaux déstabilisent et font entrer ces textes de plain-pied dans la tragédie. L'innocence se retrouve mêlée à la plus dure des violences. Dans Les fous de Bassan, la scène précédant le meurtre des deux cousines Atkins est particulièrement révélatrice de la dramatique confrontation du désir et de la violence. Ces deux pôles axiologiques sont incarnés par Nora et Stevens. Nora est associée au désir, un désir naissant, un désir d'enfant qui provoque le jeu sans pour autant passer à l'acte. Elle est porteuse d'un désir qui s'exprime avec des connotations positives : «plaisir», «se grisant », « rit », « son rire de gorge », « désir », « caresse apaisante. » (FB: 244) Face à cela, Stevens incarne la violence et la mort. Le versant sombre du désir qui provoque et charrie toute une isotopie malveillante : «menace », « cognant », « déteste », « pleure », « vociférante », « fruste », « hystérique ». (FB: 244) Ici s'incarne le couple Éros et Thanatos, l'Amour et la Mort, dualité récurrente dans les romans: « Je suis la vie et la mort inextricablement liées. Vois comme je suis douce-amère. » $(K: 161)$

Face à l'impossibilité d'arriver à un terrain d'entente sexuel, la femme se trouve obligée de dénoncer la violence masculine. Pour cela, elle a recours au langage masculin dont la violence va servir d'outil de dénonciation. C'est en effet par la langue que la femme va amorcer la critique de la violence sexuelle subie. 


\section{Une dénonciation implicite de cette violence : analyse phrastique et lexicale}

À l'image des héroïnes qui oscillent continuellement entre paraître et être, indicible et diction, pudeur et fureur, le texte avance en reculant. La dislocation atteint les instances énonciatrices ainsi que les propos de ces femmes qui hésitent entre pudeur et fureur, entre se taire et s'exprimer. Par le recours aux structures paratactiques, aux phrases segmentées aux parallélismes et autres disjonctions, les femmes procrastinent volontairement pour diverses raisons. Les phrases segmentées vont entre autres permettre de présenter des pôles opposés et les structures à parallélismes permettront quant à elles de mettre en relief certains termes ayant une grande importance sémantique. Ces structures servent la dénonciation de la violence masculine : «N'ayant plus à craindre d'affront de la part de son mari, Felicity aborde l'âge d'être grand-mère comme quelqu'un qui commence à vivre. » (FB:36)

Cette phrase segmentée présente une inversion de l'ordre syntagmatique des propositions. La subordonnée est antéposée, ce qui segmente la phrase en deux. Cette segmentation centrale permet d'organiser et d'opposer les contenus sémantiques des deux propositions autour d'une scission sémantique centrale. La subordonnée, en étant antéposée, évite une progression liée de la phrase qui aurait induit un mouvement de la phrase vers une fermeture, une fin. La subordonnée antéposée aborde le pôle masculin qui, dans l'œuvre, est une instance de fermeture et d'emprisonnement. À l'inverse, la proposition principale est dédiée au pôle féminin qui se veut résolument ouvert. Ainsi, en présentant tout d'abord le pôle masculin fermé et en lui opposant le pôle féminin ouvert, la phrase s'achève sur un mouvement ascendant, symbole d'une nouvelle naissance et d'une nouvelle vie pour la femme. La segmentation de cette phrase permet l'évolution en deux temps distincts. Il y a, tout d'abord, un temps du mâle/mal connoté par l'angoisse et le négatif mais qui s'achève. On peut souligner la tournure négative de la proposition subordonnée et relever la présence d'une isotopie négative : «craindre », « affront », et pourquoi ne pas ajouter «mari » qui est porteur des valeurs patriarcales. À l'opposé, se développe un temps féminin de l'accession à soi marqué par la sagesse et la sérénité. Le prénom de la grand-mère, «Felicity », évoque déjà le terme «félicité » qui désigne un état de bien-être. C'est ensuite toute une isotopie positive qui est mise en place pour sous-tendre l'impression de sagesse et de bien-être. Le verbe « aborde » est connoté positivement ici, il signifie une ouverture à l'extériorité ainsi que l'espoir. Le verbe « être » marque quant à lui la fin de l'ère du paraître et l'entrée de plain-pied dans l'être. Le verbe « commence » évoque lui aussi une nouvelle naissance, voire 
une naissance puisqu'il n'est pas dit « recommence ». Le terme « grand-mère » évoque un temps de la sagesse et de la sérénité. Enfin, le verbe « vivre » est mis en relief du fait de sa position finale et ouvre définitivement sur une nouvelle histoire, une nouvelle vie.

La forme fait sens, c'est une « forme-sens » selon l'expression d'Henri Meschonnic. L'ordre inversé permet de faire affleurer textuellement l'opposition entre hommes et femmes. Le texte devient l'outil d'une mise en scène dramatique des discours féminins.

\section{Une quête de sensualité}

Après avoir dénoncé la violence sexuelle masculine, I'héroïne hébertienne doit se créer un «langage-femme » lui permettant enfin de dire son désir sans qu'il ne soit faussé par un lexique mâle. La société patriarcale ébranlée et au bord de l'effondrement, la femme peut à loisir imaginer un nouveau schéma relationnel qui ne serait plus un combat permanent. Ce nouveau schéma homme-femme imaginé par les femmes n'étant cependant pas réalisé, force est de constater que les héroïnes hébertiennes, sans langue, doivent aller jusqu'aux confins de la parole pour dire leurs désirs à travers le silence ou le cri.

\section{Le désir d'un autre contact homme-femme}

Refuser de s'insérer dans les codes et valeurs de l'ordre patriarcal en place est la marque d'une affirmation de soi et de la prise de conscience que, pour « se réaliser », il ne faut plus passer par la voix d'autrui, mais acquérir sa propre langue, son propre ordre. Il ne semble pas pouvoir y avoir de lieu commun d'énonciation entre hommes et femmes, ce qui entraîne la naissance d'une écriture spécifique qui ne se laisserait pas englober dans un code esthétique mais le déborderait. L'enjeu n'est pas seulement de communiquer, mais de rééquilibrer la société en ouvrant la voie au désir féminin. Les femmes font violence à la langue pour la plier à leur voix et à l'expression de leurs désirs. Scène d'une lutte à mort entre le sujet masculin qui cherche encore à exercer sa maîtrise sur le monde et cette altérité qui fait irruption dans l'écriture, le roman devient le lieu d'une violence innommable, apocalyptique, qui disloque et désorganise l'énonciation ; l'écriture et le texte pour les féminiser. Rythme syncopé, syntaxe simplifiée, affleurement de la jouissance énonciative : autant de signes discursifs qui témoignent de cette volonté de puissance sur la parole. Cette voix féminine qui est déjà présente dans les textes doit à présent, pour s'ajuster parfaitement aux femmes, se transforme en un «langage-femme ». Cette diction féminine passe 
donc par une énonciation hachée et violente qui tend à déconstruire et à détruire la syntaxe jusque-là codifiée et utilisée par les hommes. La désorganisation de cette linéarité masculine symbolise le démantèlement de l'institution patriarcale souhaité et entamé par les femmes et passe par une véritable émasculation de la structure phrastique. Le désir féminin prend forme, il invente une nouvelle grammaire qui se veut la conjonction du signifié (le sentiment) et du signifiant (le mot). La parole et l'écriture épousent le mouvement pulsionnel de l'âme féminine. Mouvement qui se concrétise par des phrases nominales, diamants bruts de désir : « Le poids d'un homme sur moi. Son poil de bête noire. Son sexe dur comme une arme. » ( $K: 156)$ Le désir submerge la diction qui se trouve soumise au surgissement des sentiments féminins. Hésitant entre pudeur et fureur, ce désir entraîne le texte dans sa folie et sa jubilation jusqu'à le mener à bout de souffle et de mots.

Pour transmettre et donner à voir leur désir, les personnages féminins recourent ici à une syntaxe du ressenti dont l'objectif est de faire sentir aux autres ce qu'euxmêmes ressentent : leur désir, l'urgence de vivre, le plaisir de dire. Quel procédé pourrait mieux rendre textuellement la fureur et la jubilation que l'énumération ? L'énumération permet de créer une impression de foisonnement. L'énumération ne réclame aucune fin puisque c'est sa propagation même qui fait sens au fur et à mesure. Comme le souligne Jean-Louis Major, « [I]'énumération ne s'arrête que par accident, par l'intervention d'une volonté. Si le premier mot en paraît souvent involontaire ou « donné », son arrêt, au contraire, révèle un choix. » (Major, 1976 : 75)

Le style télégraphique, par la juxtaposition d'un grand nombre de phrases simples et courtes, voire lapidaires qui vont droit au but, permet lui aussi de tracer « I'urgence de vivre » des femmes. Cette écriture télégraphique s'établit dans la longueur et fait sens par sa progression pointilliste et épurée.

Mes jupes sont pleines de boue. Mon corsage est décousu. Nous courons tous les deux. À perdre haleine. Sur la grève mouillée. Tombons dans les joncs. Les petites flaques d'eau vertes qui éclatent sous notre poids. Les algues visqueuses rouges, jaunes. Des fougères de mer, dessinées sur notre peau. Antoine Tassy mon mari. Ah ! si les domestiques ou les villageois... Nous sommes deux enfants sauvages. Donnons-nous la main. Embrassons-nous sur la bouche. À perdre le souffle. ( $K$ : 84-85) 
Il n'y a plus de revendication ou de désir de changer et de bouleverser l'ordre stagnant, seulement l'envie d'être, l'urgence du désir physique à l'état « sauvage » se manifestant violemment et passionnément. La pulsation du désir atteint l'énonciation et le texte. Imitant le rythme fou d'un cœur qui s'emballe à cause du désir et de la course, le texte est haché, chaque phrase est une pulsation qui, à peine prononcée, est réalisée, et qui, à peine réalisée, est immédiatement prolongée par une autre pulsation, et ainsi de suite, dans un mouvement vertigineux. Le vertige est dû à la progression croissante du désir qui ira jusqu'à l'explosion. C'est la quête de ce point culminant qui sous-tend l'écriture de la fureur qui nous intéresse. Telle une protase passionnelle, le style télégraphique donne à lire le mouvement ascensionnel du désir. Ce style discontinu et sec auquel ont recours les narratrices provoque la jubilation de la parole du désir qui fut si longue à advenir.

Les phrases nominales et infinitives viennent appuyer cette jubilation tout en laissant planer un doute sur l'identité du sujet émetteur du désir. Par le biais des phrases nominales et infinitives, les narratrices s'inscrivent et inscrivent leurs désirs, dans un statut indifférencié. Le modèle patriarcal toujours en place n'acceptant pas la diction féminine du désir, la femme exprimera un désir en apparence neutre en s'effaçant de la fonction de sujet-émetteur-énonciateur. Impossible donc en apparence d'affirmer un quelconque sexe du désir dans ces phrases nominales. « L'odorat part en flèche, trouve sa proie. La découvre et la reconnaît. Lui fait fête. Accueille l'odeur de l'assassin. La sueur et l'angoisse, le goût fade du sang. Ton odeur, mon amour, ce relent fauve. » $(K: 212)$ La narratrice joue ici de la mixité des genres pour dérouter le lecteur et les témoins. Elle cherche à mêler les deux pôles du désir, prenant ici la forme sensorielle de l'odorat, en les faisant se rapprocher d'une forme d'androgynie. Il y a tentative ici de démasculiniser et déféminiser les personnages afin de ne plus pouvoir identifier la source et le sexe du désir. Cette quête de neutralité et d'androgynie tend à leurrer la censure textuelle patriarcale qui refuse la diction du désir par les femmes.

Si le sujet n'est pas toujours clair, c'est aussi parce que les femmes rêvent d'atteindre une société plus égalitaire où le sexe ne définirait plus ce que l'on peut ou ne peut pas dire. En effaçant le sujet, la narratrice certes voile son désir et cela relève de la pudeur mais, simultanément, elle tire le texte vers une androgynie émasculatrice. C'est vers ce nouvel ordre que tend Nora qui revendique cette égalité entre les sexes et rêve d'harmonie et de communication égalitaire : «[I]l serait si facile de s'entendre comme deux personnes, égales entre elles, dans l'égalité de leur désir. » ( $F B: 127)$ Elle ouvre la voie à une nouvelle génération de personnages de fictions 
féminins et imagine un avenir positif, une égalité possible entre hommes et femmes. Telle une « féministe en herbe », Nora conçoit les relations selon un pacte d'échange et de plaisir :

Sans nous déshabiller, encombrés de nos vêtements, sans même nous tenir la main, les garçons et moi, nous communiquons déjà, par le frisson, par la fièvre cachée, tandis que nos visages seuls découverts, avec nos mains nues, sourient et se colorent de lumière. » (FB:118-119)

Le désir est dit dans toute sa nudité et son impudeur. Au désir passionnel et pulsionnel correspond une écriture et une parole passionnelles et pulsionnelles. Ce qu'Elisabeth désigne ainsi : «Tout un langage incohérent, haletant, impudent et cru. » ( $K: 101)$

\section{La diction du désir, entre silence et cri}

L'écriture féminine, la « texture féminine » (Smart, $2003: 25)$ selon l'expression utilisée par Patricia Smart, est insaisissable par définition ; elle résiste aux codifications et de fait s'extraie des schémas de lecture et de critique habituels qui se fondent uniquement sur la signification. Ce n'est qu'en se mettant à l'écoute de ce qui semble ne pas signifier dans le texte, de cette «foule de riens féminins » (Conan dans Smart, 2003 : 25), que le lecteur et le critique pourront saisir cette texture féminine. Le recentrement de l'énonciation sur le ressenti, provoque de nombreuses ruptures textuelles dans les romans d'Anne Hébert. Si la dislocation des phrases est signifiante, il convient aussi de reconnaître aux brèches qu'elle engendre un grand « pouvoir de germination ». (Oore, 1997 : 386) Ainsi, dans ces creux apparents que sont le silence et les suspensions de la narration, le texte continue de dire. Tout ce que le roman aborde, le silence le redouble : «Le silence double le temps, lui donne sa mesure impitoyable. » ( $K: 243)$ Dans cette zone d'ombre qui rayonne sur le texte, se cristallisent les passions, les rancœurs, les révoltes jusqu'à ce que le silence ne puisse plus contenir autant de colère. Ce n'est que là, à ce point de rupture extrême, lorsque le silence devient sonore, que la déchirure se produit et que le cri fuse. Perte de tout contrôle et de toute retenue, le cri lacère les romans, provoque à son tour des failles et des éclairs de fulgurance signifiante. Le cri, tel qu'Anne Hébert le conçoit, n'est plus seulement un son perçant, il est parole d'exultation et d'exaltation. Cri de révolte qui veut ébranler la fixité d'un monde, cri de désir d'un corps fiévreux, le cri à l'instar du silence n'est pas forcément un échec de la parole, mais bien une parole inarticulée, ultime, pure et tranchante. Les femmes recourent au cri comme à un couteau pour poignarder le texte, ce corps mâle, cet ordre patriarcal qui les opprime. Le cri est violence envers le texte mais il est aussi inscrit au sein du texte 
et se manifeste par le corps. Les romans développent une gestuelle du cri et de ses manifestations tant physiques que physiologiques. Ce retour aux extrêmes confirme I'incapacité de la langue à dire la féminité, à dire l'être-femme et le ressenti-femme. La parole prononcée, écrite, académique paraît finalement, malgré sa dislocation, encore trop neutre, trop timide, trop timorée et seul un certain extrémisme langagier semble pouvoir traduire le désir féminin.

\section{Les points de suspension}

Inscrit dans la parole ou la non-parole des personnages, inscrit dans les brèches textuelles et sémantiques, le silence a pour principe habituel de ne pas être visible. Pourtant, il prend forme dans le texte et signifie sa présence. Il fait signe, il est signe. Il attire l'attention du lecteur en se manifestant par des points de suspension : «... ». Le lecteur comprend rapidement que derrière ces points suspensifs, se dissimule le mot, le sens, la clef de l'énigme comme autant d'éléments suspendus temporairement. Comme le note Gaston Bachelard, « les points de suspension [...] tiennent en suspens ce qui ne doit pas être dit explicitement. » (Bachelard, 1978 : 51) Dans les romans, ils sont donc une marque très récurrente et cachent l'indicible naissance du désir féminin. Souvent placés stratégiquement, ils occultent et insistent simultanément, et produisent un effet de redoublement sémantique. La femme peut être désirée mais pas désirante, ce qui provoque la création d'un masque de pudeur à poser sur ses paroles. Son désir n'a pas le droit de se dire ou de s'écrire autrement que sous forme implicite et par l'usage des points de suspension : «Être retournée par le son de cette voix, être remuée, fouillée, ouverte par le son de cette voix, comme si... » ( $K: 208)$ De plus, c'est un désir naissant qui cherche à s'exprimer. Peu assuré, il tâtonne et reste encore pudique. Malgré son côté provocateur et ses penchants féministes, Nora maintient des barrières à même l'énonciation de ses expériences : «Non, non je ne tolèrerai pas que l'Américain me touche, ni avec ses grosses mains, ni avec sa bouche molle, ni... Mon Dieu, non. Non, non, je ne le tolèrerai pas. » ( $F B: 119)$ La jeune fille n'ose pas encore tout dire, c'est la preuve de sa réticence à grandir trop vite. Elle joue mais ne veut pas aller plus loin avec cet inconnu maintenu par la parole dans une indistinction. D'autres héroïnes se trouvent déchirées entre leur pudeur enfantine et leur curiosité. «Aurélie, il faudrait que je te parle pourtant. Comment faire ? Je voudrais savoir... Les garçons... Les garçons... » (K: 64) Entre envie et crainte, l'ambivalence des héroïnes ne trouve pas de résolution définitive. 
Cependant, au fil des prises de parole chargées de désir, le ton et le choix des mots semblent s'affermir, ne laissant plus de doute possible sur le mot passé sous silence : «Une sorte de rituel entre nous. Chaque fois que nous sommes ensemble dans le bois de pins et qu'il fait encore trop clair pour... Nous jouons aux gisants de pierre. Nos deux corps étendus. » $(K: 148)$ Les points de suspension retiennent l'attention du lecteur qui croit tout d'abord y voir une pause, un blanc et un silence, mais comprend enfin que ces caractères typographiques sont signifiants.

\section{Le cri}

Après avoir mûri au creux du silence, les passions cristallisées doivent se confronter au monde extérieur. Elles jaillissent sous la forme d'un cri sans concession qui se veut fulgurant, violent et passionné. Le cri apparaît comme l'expression ultime du désir féminin, il est révolte, plaisir et jouissance. Effrayant pour les autres, il est jubilation pour l'émetteur. Mais faut-il y voir une épuration parfaite ou un échec de la parole ? N'ayant pas trouvé les mots pour s'exprimer dans la langue mâle, les femmes ne trouvent-elles pas dans le cri l'incarnation de ce «langage-femme » à inventer ? Riche en sensations, le cri est la flèche ultime décochée par le désir féminin qui s'affirme une fois pour toutes sans détour. «Le silence pousse dans ma bouche comme une herbe. Tous les mots, un jour, me furent livrés. Ne trouve que ce cri. » (Fuvre poétique 1950-1990, 1993 : 110)

De plus, le désir ne se traduit que difficilement en mots. Comme le dit Patricia Smart, « le désir dépasse les limites et la mesure du langage et ne sait éclater qu'en cris insoutenables ». (Poulin dans Smart, 2003 : 273) Le désir n'est pas contrôlable, la voix qui s'essaie à le dire ne peut donc pas être stricte et figée. Le désir réclame une diction qui soit à son image, instable, heurtée et imprévisible, voix soupirante ou exaltée jusqu'à l'inarticulation fiévreuse : « J'ai tant crié. Une espèce de crécelle stridente dans ma gorge. Une mécanique terrible déclenchée. Incontrôlable. Cela n'a plus rien d'humain, m'étouffe et m'épouvante. » ( $K: 115-116)$ Le désir renvoie à un état primitif, inhumain en ce qu'il a la capacité à faire oublier tous les codes. Le désir est un torrent qui ne se soucie guère des réticences de la conscience et de la bienséance, il entraîne tout avec lui dans un mouvement des sens provoquant une perte de contrôle sur la langue et le langage. Lorsque toute parole logique est annihilée par le surgissement du désir, il reste le cri : «Dès qu'elle m'a vu dans la porte d'entrée, elle a poussé une sorte de cri, plein de mots tendres et sauvages, s'entrechoquant les uns les autres. » (FB: 74-75) «Un cri rauque s'échappe de la gorge de ma cousine, 
bientôt des paroles s'entremêlent dans sa bouche ». (FB:66) Le désir provoque une « régression compulsive vers le cri. » (Marcheix, $2005: 460)$ Le cri est aussi cri orgasmique, cri de plaisir tout particulièrement chez Elisabeth : «Tu rentres entre mes cuisses, au plus profond de mon ventre. Je crie et je t'appelle, mon amour ! » ( $K$ : 208-209) Ici, la phrase exclamative vient appuyer ce cri, le redoubler.

Le cri est pulsation de souffrance, de désir et de vie ; il est « cette petite voix impérieuse qui cogne contre son cœur et qui demande la parole. » $\left(O P^{5}: 97\right)$ Avant de s'extérioriser, il se trame dans un corps. À l'état latent, alors qu'il n'est qu'une pulsation au cœur du silence, il permet aux héroïnes de prendre conscience de leur corps et de la sensibilité de celui-ci. Chaque héroïne, en fonction de son histoire, ressent cette pulsation de vie, de révolte et de désir martelant un rythme enivrant dans tout son corps, le rythme d'une émancipation en germe : «Elle allongea les jambes, ferma les yeux, feignant le calme, tandis que son cœur battait à se rompre dans sa poitrine, dans son cou, à la racine de ses poignets. » $(C B: 146)$ Le cri du désir qui assaille Catherine se manifeste par le biais d'une pulsation de son cœur en éveil qui, en se déplaçant, réveille son corps endormi. Cette pulsation interne est un éveil sensuel. Première manifestation de ce cri du corps qui veut se sentir vivre et que I'on retrouve chez Elisabeth : «Le cœur qui se déplace à grands fracas dans mon corps. Cogne à ma tempe, dans mon cou, à mon poignet. » $(K: 192)$ Le «cri sourd » qui habite ces héroïnes est le cri du désir trop longtemps contenu en raison de l'inégalité des sexes. Cette pulsation favorise la prise de conscience du corps. Cette petite voix, ce battement continu conduisent à la piste d'une possible exultation du corps. Parole ultime du désir, le cri est la rencontre entre le silence lourd de la fièvre secrète animant les corps et l'acuité sensorielle provoquée par le monde extérieur. Manifeste du «langage-femme » il est l'exemple de ce vers quoi doit tendre I'héroïne dans sa quête d'une « voix juste et belle pour chanter les noces de [la femme] avec la terre. » $(O P: 59)$

À la vie, à la mort, les relations homme-femme s'enlisent dans une violence sans borne qui atteint son apogée dans la sexualité. Dans les romans hébertiens, les hommes autant que les femmes semblent voués à reproduire des schémas ancestraux violents. Leur sexualité est un combat pour le pouvoir longtemps dominé par les hommes. Cependant, les héroïnes des Chambres de bois, de Kamouraska et des Fous de Bassan trouvent les moyens de prendre le contrôle de la parole pour enfin se libérer du joug masculin, assouvir leurs désirs et les exprimer. Cette découverte

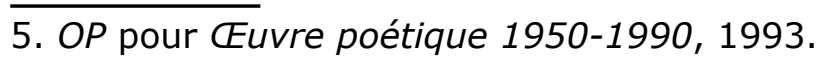


du plaisir simultanément à l'expression de leurs désirs chamboule la langue. Parole de jouissance, la voix féminine crie et s'impose contre la sexualité mortifère du mâle. 


\section{Bibliographie}

AQUIN, Hubert (1991), L'invention de la mort, Québec, Bibliothèque québécoise.

BACHELARD, Gaston (1978), L'eau et les rêves. Essai sur l'imagination de la matière, Paris, José Corti. BOISCLAIR, Isabelle (2000), «Au pays de Catherine », Les cahiers Anne Hébert, n 2 : 111-125.

COLLIN, Françoise (1976), « Polyglo(u)ssons », Les Cahiers du Grif, n 12 : 3-9.

HÉBERT, Anne (1996 [1958]), Les chambres de bois, Paris, Seuil, coll. « Points ».

HÉBERT, Anne (1997 [1970]), Kamouraska, Paris, Seuil, coll. « Points ».

HÉBERT, Anne (1982), Les fous de Bassan, Paris, Seuil.

HÉBERT, Anne (1993), CEuvre poétique 1950-1990, Montréal, Boréal.

MAJOR, Jean-Louis (1976), Anne Hébert et le miracle de la parole, Montréal, Presses de l'Université de Montréal.

MARCHEIX, Daniel (2005), Le mal d'origine. Temps et identité dans l'œuvre romanesque d'Anne Hébert, Québec, L'instant même.

OORE, Irène (1997), " Le silence dans L'enfant chargé de songes d'Anne Hébert », dans Madeleine Ducrocq-Poirier [et al.] (dir.), Anne Hébert, parcours d'une œuvre. Actes du colloque de la Sorbonne. Montréal, L'Hexagone : 383-395.

RANDALL, Marilyn (2001), « Les fous de Bassan à l'écran : lecture aberrante... éclairante ? », Les Cahiers Anne Hébert, n 3 : 103-117.

SMART, Patricia (2003), Écrire dans la maison du père : l'émergence du féminin dans la tradition littéraire du Québec, Montréal, XYZ.

TULLOCH, Elspeth (2001), « Voix, viol et réécriture symbolique : Les fous de Bassan à l'écran », Les Cahiers Anne Hébert, n 3 : 77-102. 